\section{Disfunção familiar referida pela presença de depressão materna e/ou alcoolismo na família e ocorrência de cárie dentária em crianças de dois e três anos de idade}

\section{Family dysfunction related to maternal depression and/or alcoholism in the family and dental caries in children from two to three years old}

Marcos Augusto de Andrade Souza 1

Maria Isabel Pereira Vianna 2

Maria Cristina Teixeira Cangussu 3

1-3 Departamento de Odontologia Social e Pediátrica. Faculdade de Odontologia. Universidade Federal da Bahia. Rua Araújo Pinho, 62. Canela. Salvador, BA, Brasil. CEP: 41.110-150

\begin{abstract}
Objectives: identify the association between family dysfunction related to the presence of alcoholism in the family and/or maternal depression symptoms and dental cavities/caries in children from two to three years old.

Methods: population comprised of 74 families in Salvador, Bahia. Data collection consisted of the clinical examination of the children and interviews of their mothers at home. Criteria of the World Health Organization were used to diagnose dental cavities/caries followed by disease prevalence classification. To identify symptoms of maternal depression and/or alcoholism in the family the Self-Report Questionnaire (SRQ-20) was used as well as the adapted CAGE scale, respectively. Descriptive analysis and adjusted prevalence ratio for confusion variables were estimated through non-conditional logistic regression.

Results: population's social and economic profile was homogenous. Caries/cavities in children prevalence was of $20.2 \%$. A positive association between family dysfunction and caries was determined $\left(P A_{\text {gross }}=3.06\right.$; 95\%CI: 1.07-8.75). When this model was adjusted through confusion co-variables, family leadership and maternal job situation in the last two years, an increment of the association was noted ( $P A_{\text {adjusted }}=7.35$; 95\% CI: 1.50-36.07).

Conclusions: an association between family dysfunction and dental caries in small children is suggested.
\end{abstract}

Key words Dental caries, Social adjustment, Epidemiology, Child

\section{Resumo}

Objetivos: identificar a associação entre disfunção familiar, referida pela presença de alcoolismo na família elou presença de sintomas de depressão materna e a cárie dentária em crianças de dois e três anos de idade.

Métodos: a população consistiu de 74 famílias de Salvador, Bahia. A coleta de dados compreendeu o exame bucal das crianças e a entrevista com suas mães no domicílio. Foram utilizados para o diagnóstico de cárie dentária os critérios adotados pela da Organização Mundial de Saúde, classificando posteriormente a prevalência da doença. Para a identificação de sintomas de depressão materna elou caso de alcoolismo na família foram utilizados o Self-Report Questionnaire (SRQ-20) e a escala CAGE adaptada, respectivamente. Procedeu-se a análise descritiva, e a razão de prevalência ajustada para as variáveis de confusão estimada por meio de regressão logística não-condicional.

Resultados: a população revelou-se homogênea no perfil socioeconômico. A prevalência de cárie em crianças foi de 20,2\%. Observou-se uma associação positiva entre disfunção familiar e cárie $\left(R P_{\text {bruta }}=3,06\right.$; IC95\%: 1,07-8,75). Quando este modelo foi ajustado pelas covariáveis de confusão, chefia da família e situação de trabalho materno nos últimos dois anos, detectouse incremento da associação $\left(R P_{\text {ajustada }}=7,35\right.$; $I C 95 \%$ : 1,50-36,07).

Conclusões: existe uma associação entre disfunção familiar e ocorrência de cárie dentária em crianças de dois e três anos de idade.

Palavras-chave Cárie dentária, Ajustamento social, Epidemiologia, Criança 


\section{Introdução}

O estudo das condições de saúde bucal de populações humanas, historicamente, privilegiou aspectos biológicos em relação à ocorrência da cárie dental. Entretanto, nas últimas décadas, pesquisas têm buscado relacionar os modos de vida de determinados grupos, envolvendo aspectos como o nível de renda, a escolaridade e os padrões de consumo, com a situação de saúde bucal. ${ }^{1}$ Alguns estudos têm apontado, também, para a importância de fatores associados com o estilo de vida e padrões de comportamento familiar e individual, como decisivos na conformação da epidemiologia dessa enfermidade. 2,3

Estudos epidemiológicos recentes têm apresentado uma melhoria dos índices desta doença na faixa etária escolar, fenômeno de caráter mundial,4,5 atribuído à difusão do uso de fluoretos nas últimas décadas, expansão dos serviços de saúde, entre outros fatores. 6 Particularmente, em pré-escolares, esta redução se apresenta com uma magnitude menor do que a observada no grupo escolar. 5 Podem ser identificados, como potenciais fatores associados à ocorrência da doença nesse grupo etário, o padrão de aleitamento, a época de introdução do desmame, hábitos dietéticos; o estabelecimento de práticas de higiene bucal quando da erupção das primeiras unidades dentárias e a contaminação precoce com os microorganismos cariogênicos. ${ }^{7}$ Cada um desses fatores envolve decisões que podem agir de modo a evitar ou abreviar a instalação da cárie dental e também influenciar decisivamente na intensidade e severidade da sua evolução.

Na maioria das vezes, a mãe exerce o papel principal no controle dessas variáveis, tendo como cenário o ambiente familiar, que, de certa forma, propicia condições favoráveis ou não para que todos os cuidados necessários para um bom desenvolvimento emocional, cognitivo e físico da criança sejam implementados.

Neste sentido, é pertinente a construção de modelos explicativos que contemplem, além dos fatores clássicos associados à ocorrência de cárie em crianças de idade pré-escolar, os elementos que podem estar determinando a maior ou menor exposição das crianças aos referidos fatores, particularmente àqueles relacionados com o ambiente familiar.

A ocorrência de alcoolismo na família e/ou depressão materna pode comprometer significativamente a capacidade da mãe de prover cuidados para crianças na idade pré-escolar. Várias pesquisas têm apresentado associação significante entre disfunções familiares e alguns desfechos negativos para a saúde de um modo geral. 8,9 Nessa faixa etária, esse tema se torna ainda mais relevante, considerando-se a maior vulnerabilidade aos distúrbios familiares, 1,9 visto que, existe um grau de dependência significativo entre as crianças e os seus cuidadores, sendo estes, na maioria das vezes, a mãe. 10

Existem esforços de serviços de saúde, no contexto do Programa de Saúde da Família, na tentativa de identificar famílias sujeitas às situações especiais de risco para saúde bucal, tendo, como indicadores, o risco familiar e individual. ${ }^{11}$ Este conhecimento poderá subsidiar a identificação desses casos, de forma a contribuir para ações de planejamento e programação da atenção à saúde bucal no nível local.

Assim, o objetivo deste trabalho foi identificar uma possível associação entre disfunção familiar, referida pela presença de alcoolismo na família e/ou presença de sintomas de depressão materna e a ocorrência de cárie dentária em crianças de dois e três anos de idade em microárea do município de Salvador, Bahia.

\section{Métodos}

Trata-se de um estudo de corte transversal, do qual participaram crianças de dois e três anos de idade e suas respectivas mães, residentes em uma microárea localizada no bairro do Garcia, em Salvador, Bahia, Brasil. A região, definida por conveniência, compreende um conjunto de 10 setores censitários urbanos. Dentro da área delimitada não houve sorteio amostral. O estudo, por sua natureza exploratória, teve caráter censitário, identificando-se, mediante visita domiciliar, as famílias que tinham, entre os seus membros, crianças na faixa etária estabelecida. Para compor a população de estudo foram incluídas as crianças que permitiram a realização do exame após autorização da mãe, cuja concordância em participar se deu voluntariamente e foi expressa através da assinatura de termo de consentimento livre e esclarecido. Os critérios de exclusão consistiram em crianças com aversão ao exame bucal, crianças com enfermidades sistêmicas e/ou portadoras de anomalias na dentição. A seleção da área de estudo deveu-se à existência de serviços de saúde bucal, públicos e filantrópicos, para os quais foi possível referenciar as crianças com necessidades de tratamento, bem como a relativa similaridade de natureza socioeconômico-sanitária e cultural da população residente.

A coleta de dados compreendeu o exame clínico 
das crianças para avaliação da cárie dentária na dentição decídua (condição da coroa), utilizando-se o índice ceo-d (média de dentes decíduos cariados, extraídos e obturados numa população) ${ }^{12}$ e a entrevista das suas respectivas mães, através da aplicação de questionário estruturado. Procedeu-se o treinamento da equipe, composta por um cirugião-dentista e sete estudantes de odontologia (três examinadores e entrevistadores e quatro anotadores), através da realização de um estudo-piloto. Na análise de concordância inter-examinadores para o ceo-d obtiveram-se índices Kappa que variaram de 0,95 a 0,99. O exame clínico foi realizado no domicílio, em ambiente externo, com iluminação natural, sem secagem ou escovação prévia dos dentes, utilizandose abaixador de língua, estando a criança em posição sentada.

Em seguida, ou em data agendada, foram realizadas as entrevistas com as mães. O instrumento elaborado estava dividido em seções que envolviam: a) identificação, dados socioeconômicos e demográficos e condições gerais de saúde materna; b) dados relativos à saúde bucal na família (hábitos, exposição ao flúor, acesso a assistência); c) a escala CAGE adaptada, 10 e d) o Self-Report Questionnaire (SRQ-20), instrumento desenhado pela Organização Mundial de Saúde para a detecção de morbidade psiquiátrica na população.13 A escala CAGE é um teste de triagem auto-informativo para alcoolismo que compreende quatro questões, sendo considerado positivo quando o resultado apresenta duas ou mais respostas afirmativas. Neste estudo, o procedimento de aplicação dessa escala foi adaptado, considerando-se as respostas dadas por uma informantechave, a mãe, referindo-se a todos os moradores da casa.10 O Self-Report Questionnaire (SRQ-20) é composto por vinte questões que têm duas possibilidades de resposta ( $\operatorname{sim} /$ não) e foram desenhadas para abordar sintomas emocionais e físicos associados a quadros psiquiátricos. Conforme o indicador, morbidade psiquiátrica significativa está associada à presença de oito ou mais desses sintomas. Neste estudo, levou-se em consideração, especificamente, sintomas depressivos maternos expressos pela resposta afirmativa às questões relacionadas ao sintoma.

Com base no questionário, foram selecionadas as seguintes covariáveis para caracterização da população de estudo: Bloco I) composição e condições socioeconômicas e sanitárias da família: chefia da família; presença de companheiro; moradores do domicílio menor que 5; salário do chefe de família acima de um salário mínimo; saneamento básico; presença de banheiro. Bloco II) condições maternas em relação a saúde, escolaridade e inserção no trabalho: enfermidade materna nos últimos dois anos; escolaridade da mãe; história de trabalho materno nos últimos dois anos; carga horária do trabalho da mãe; situação atual do trabalho materno. Bloco III) condições relacionadas à saúde bucal da família: consumo de açúcar; número de escovas por domicílio; uso de pasta de dente com flúor; freqüência de escovação referida; ida ao dentista no último ano. A variável dependente foi a cárie dentária, medida através do índice ceo-d, considerando-se para a análise as categorias ceo- $\mathrm{d}=0$ e ceo-d $>0$. Como variável independente principal, utilizou-se um indicador de disfunção familiar, construído neste estudo a partir dos resultados da escala CAGE adaptada e do SRQ-20, conformandose as seguintes categorias: inexistência de caso de alcoolismo na família e de sintomas de depressão materna e ocorrência de caso de alcoolismo na família e/ou sintomas de depressão materna. Variáveis potenciais de confusão foram: chefia da família, escolaridade da mãe e história de trabalho materno nos últimos dois anos.

Procedeu-se a análise descritiva das covariáveis de interesse de acordo com a exposição, bem como foram obtidas as medidas de ocorrência do efeito e da exposição. Para testar a hipótese de associação entre disfunção familiar e cárie em crianças de dois e três anos, razão de prevalência (RP), ajustados para as variáveis de confusão, foram estimados por meio de regressão logística não-condicional e a inferência estatística baseou-se em Intervalos de Confiança a 95\% (IC95\%), calculados pelo Wald test. A seleção das variáveis para modelagem baseou-se no modelo teórico, na literatura e nos resultados da análise estratificada. $\mathrm{Na}$ análise de regressão logística, utilizou-se o procedimento backward. Variáveis de confusão foram as que, ao serem retiradas do modelo saturado, produziram alterações de pelo menos $20 \%$, na medida da associação principal, ou na amplitude de seu respectivo intervalo de confiança. Para a criação do banco de dados, utilizou-se o Epi-info, versão 6.04 e a análise foi realizada no SAS versão 8.1 .

O protocolo desta pesquisa foi aprovado pelo Comitê de Ética do Instituto de Saúde Coletiva da Universidade Federal da Bahia, em 2 de junho de 2003.

\section{Resultados}

Do total de 105 famílias que tinham entre os seus membros crianças na faixa etária estabelecida, 74 
Tabela 1

Composição e condições socioeconômico-sanitárias da família.

\begin{tabular}{|c|c|c|c|c|}
\hline \multirow[t]{3}{*}{ Variáveis } & \multicolumn{4}{|c|}{ Depressão materna e/ou alcoolismo na família } \\
\hline & \multicolumn{2}{|c|}{ Expostos } & \multicolumn{2}{|c|}{ Não-expostos } \\
\hline & $\mathrm{N}$ & $\%$ & $\mathrm{~N}$ & $\%$ \\
\hline \multicolumn{5}{|l|}{ Chefe de família } \\
\hline Pai & 32 & 91,4 & 34 & 89,5 \\
\hline Mãe ou outro & 3 & 8,6 & 4 & 10,5 \\
\hline \multicolumn{5}{|l|}{ Presença de companheiro* } \\
\hline Sim & 24 & 68,6 & 29 & 74,4 \\
\hline Não & 11 & 31,4 & 10 & 25,6 \\
\hline \multicolumn{5}{|l|}{ Salário do chefe de família } \\
\hline Mais que 1salário mínimo & 35 & 100,0 & 39 & 100,0 \\
\hline Até 1 salário mínimo & -- & -- & -- & -- \\
\hline \multicolumn{5}{|l|}{ Saneamento } \\
\hline Adequado & 35 & 100,0 & 39 & 100,0 \\
\hline Inadequado & -- & -- & -- & -- \\
\hline \multicolumn{5}{|l|}{ Possui banheiro } \\
\hline Sim & 34 & 100,0 & 38 & 100,0 \\
\hline Não & -- & -- & -- & -- \\
\hline \multicolumn{5}{|c|}{ Número moradores por domicílio } \\
\hline Até 5 & 26 & 74,3 & 29 & 74,4 \\
\hline$>5$ & 9 & 25,7 & 10 & 25,6 \\
\hline
\end{tabular}

${ }^{*} p<, 0,05$.

atenderam plenamente aos critérios de inclusão, constituindo-se a população do estudo de 16 crianças com dois anos de idade $(21,6 \%)$ e 58 com três anos $(78,4 \%)$, constituindo uma perda de aproximadamente $30 \%$.

O percentual de crianças com cárie foi de $20,2 \%$, sendo de $1,3 \%$ aos dois anos e de $18,9 \%$ na idade de três anos. O ceo-d da população estudada foi de 0,72 , com um desvio padrão de 1,8, apresentando a seguinte composição percentual: o componente "cariado" foi responsável por 93,3\% do índice, o "obturado" representou $6,7 \%$ do ceo-d e não foi identificado nenhum dente extraído por cárie. Em relação à variável independente, observou-se que $47,3 \%$ das crianças estavam expostas à disfunção familiar, ou seja, suas mães apresentavam sintomas depressivos e/ou havia indícios de alcoolismo na família (Tabela 1).

Considerando-se a caracterização da população de estudo de acordo com a exposição, observou-se, de uma maneira geral, alto grau de homogeneidade entre expostos e não-expostos, quando consideradas as covariáveis sócio-demográficas. Conforme os dados apresentados na Tabela 1, constatou-se que a maioria das famílias é chefiada pelo pai; verifica-se a presença de companheiro e, no domicílio, residem até cinco moradores, não havendo diferenças entre expostos e não-expostos. Quanto às variáveis referentes à renda e saneamento, a totalidade das mães referiu que o salário do chefe é superior a um salário mínimo e que a família dispõe de condições adequadas de saneamento.

As variáveis que descrevem as condições maternas em relação à saúde, escolaridade e inserção no trabalho estão agrupadas na Tabela 2. No que se refere à história de saúde materna, os dados mostram que a maioria das mães referiu a inexistência de enfermidade nos últimos dois anos, não havendo diferenças entre expostos $(85,7 \%)$ e não-expostos $(89,7 \%)$. Em se tratando da escolaridade materna, a proporção de mães com apenas o primeiro grau foi minoritária e se distribuiu de forma semelhante em relação à variável independente principal, ou seja, $48,6 \%$ entre expostos e $48,7 \%$ entre não-expostos. Quanto aos aspectos relacionados com a inserção no trabalho, houve também grande uniformidade entre 
Condições maternas em relação à saúde, escolaridade, inserção no trabalho e condições de saúde bucal na família.

\begin{tabular}{|c|c|c|c|c|}
\hline \multirow[t]{3}{*}{ Variáveis } & \multicolumn{4}{|c|}{ Depressão materna e/ou alcoolismo na família } \\
\hline & \multicolumn{2}{|c|}{ Expostos } & \multicolumn{2}{|c|}{ Não-expostos } \\
\hline & $\mathrm{N}$ & $\%$ & $\mathrm{~N}$ & $\%$ \\
\hline \multicolumn{5}{|l|}{ Enfermidade materna últimos dois anos } \\
\hline Não & 30 & 85,7 & 34 & 89,7 \\
\hline Sim & 5 & 14,3 & 4 & 10,3 \\
\hline \multicolumn{5}{|l|}{ Escolaridade da mãe } \\
\hline Cursando $2^{\circ}$ grau e + & 18 & 51,4 & 20 & 51,3 \\
\hline $1^{\circ}$ grau completo & 17 & 48,6 & 19 & 48,7 \\
\hline \multicolumn{5}{|c|}{ História trabalho materno nos últimos dois anos* } \\
\hline Trabalhou & 30 & 85,7 & 23 & 60,5 \\
\hline Não trabalhou & 5 & 14,3 & 15 & 39,5 \\
\hline \multicolumn{5}{|l|}{ Carga horária do trabalho da mãe* } \\
\hline Até 30 horas semanais & 9 & 33,3 & 7 & 22,6 \\
\hline+30 horas semanais & 18 & 66,7 & 24 & 77,4 \\
\hline \multicolumn{5}{|l|}{ Consumo açúcar por família } \\
\hline$<6 \mathrm{~kg} / \mathrm{mês}$ & 22 & 62,9 & 23 & 59,0 \\
\hline$>$ ou $=6 \mathrm{~kg} / \mathrm{mês}$ & 13 & 37,1 & 16 & 41,0 \\
\hline \multicolumn{5}{|l|}{ Número de escovas por domicílio* } \\
\hline$>\mathrm{ou}=4$ escovas & 23 & 65,7 & 31 & 79,5 \\
\hline$<4$ escovas & 12 & 34,3 & 8 & 20,5 \\
\hline \multicolumn{5}{|l|}{ Pasta de dente com flúor } \\
\hline Sim & 35 & 100,0 & 39 & 100,0 \\
\hline Não & -- & -- & -- & -- \\
\hline \multicolumn{5}{|l|}{ Freqüência de escovação da criança* } \\
\hline 3 vezes e $+/$ dia & 12 & 34,3 & 10 & 25,6 \\
\hline$<3$ vezes/dia & 23 & 65,7 & 29 & 74,4 \\
\hline \multicolumn{5}{|l|}{ Ida ao dentista no último ano criança* } \\
\hline Sim & 9 & 25,7 & 5 & 12,8 \\
\hline Não & 26 & 74,3 & 34 & 87,2 \\
\hline \multicolumn{5}{|l|}{ Situação atual do trabalho materno } \\
\hline Outro & 24 & 70,6 & 20 & 55,6 \\
\hline Desempregado/Procura de emprego & 10 & 29,4 & 16 & 44,4 \\
\hline
\end{tabular}

os grupos. A maioria da população referiu a condição outro (dona de casa/ não precisa/ marido não deixa e está trabalhando) em relação à situação atual de trabalho e carga horária semanal superior a trinta horas. Quanto à história de trabalho materno, embora em ambos os grupos a maioria tenha referido que trabalhou nos últimos dois anos, a diferença entre as categorias foi maior no grupo dos expostos (Tabela 2).
As características das famílias quanto aos aspectos relacionados à saúde bucal também tiveram distribuição semelhante entre expostos e nãoexpostos. A maioria das mães referiu consumir mais de $6 \mathrm{~kg}$ de açúcar por mês, dispor de quatro ou mais escovas em média por domicílio e escovar os dentes das crianças em idade pré-escolar menos de três vezes ao dia. Uma minoria respondeu afirmativa- 
Tabela 3

Razão de prevalência bruta e ajustada e os respectivos intervalos de confiança a $95 \%$, para associação entre a depressão materna e/ou alcoolismo na família e a cárie dentária em crianças de dois e três anos de idade, obtidas com regressão logística.

\begin{tabular}{lcc}
\hline Modelos & RP & IC $95 \%$ \\
\hline $\begin{array}{l}\text { Modelo 1 - Medida Bruta } \\
\text { Não- expostos }\end{array}$ & 1,00 \\
$\quad$ Expostos & 3,06 & $(1,07-8,75)$ \\
Modelo 2 - Medida Ajustada (chefe de família e \\
história de trabalho materno nos últimos dois anos) \\
$\quad$ Não-expostos \\
Expostos \\
$\quad 7,00$ \\
\end{tabular}

mente à questão relativa à ida ao dentista no último ano e a totalidade das mães referiu o uso de creme dental com flúor (Tabela 2).

Observa-se a associação positiva entre a ocorrência de cárie dentária e a disfunção familiar na família. Os resultados da análise logística apresentados na Tabela 3 mostraram uma associação positiva entre disfunção familiar e cárie dentária em crianças de dois e três anos de idade $\left(\mathrm{RP}_{\text {bruta }}=3,06\right.$; IC95\%: 1,07-8,75). Quando este modelo foi ajustado pelas covariáveis de confusão, chefia da família e situação de trabalho materno nos últimos dois anos, observou-se um aumento da medida pontual e ampliação do intervalo de confiança $\left(\mathrm{RP}_{\text {ajustada }}=7,35\right.$; IC95\%: 1,50-36,07).

\section{Discussão}

Os resultados encontrados sugerem que existe, na população estudada, associação entre disfunção familiar, resultante da presença de sintomas de depressão na mãe e/ou de caso de alcoolismo na família, e a ocorrência de cárie dentária em crianças de dois e três anos de idade. A condição da mãe como chefe da família e o fato dessa não trabalhar fora nos últimos dois anos foram atributos que, após o ajuste, aumentaram a força da associação observada. Entretanto, esse aumento foi acompanhado de uma maior amplitude do intervalo de confiança, provavelmente devido ao pequeno número de famílias que compuseram a população de estudo.

Considerando-se as medidas de ocorrência da cárie dentária, pode-se dizer que a prevalência encontrada é compatível com resultados de outros estudos envolvendo a mesma faixa etária.3,14
Observou-se um aumento da prevalência de cárie nas idades de dois e três anos, o que está de acordo com a literatura, que refere o aumento da prevalência cárie com a idade em função do maior tempo de exposição aos fatores de risco presentes no meio bucal. ${ }^{14} \mathrm{O}$ índice ceo-d encontrado foi semelhante ao observado por Barros et al., 15 em um estudo que avaliou as condições de saúde bucal de crianças na faixa etária de 0-30 meses de idade, em 20 creches de Salvador, no qual a prevalência de lesões dentárias cavitadas foi de $17,6 \%$ e o índice ceo-d de 0,23 até dois anos e 0,76 para crianças de 25 a 30 meses de idade. No estudo realizado por Peres et $a l ., 3$ envolvendo crianças de 0 a 36 meses, na cidade de Pelotas, utilizando os mesmos critérios diagnósticos, encontraram-se índices ceo-d de 0,27 e de 1,40 para as idades de dois e três anos, respectivamente.

Embora não tenham sido identificados estudos que focalizem a hipótese do presente trabalho, pesquisas envolvendo a associação entre fatores desestruturantes do contexto familiar e problemas de saúde ou de desenvolvimento na infância têm sido desenvolvidos 10,15-20 e reforçam a idéia de que, para além das clássicas explicações centradas na biologia ou no estudo de variáveis socioeconômicas, como escolaridade e renda, ou ainda de variáveis relacionadas com padrões de comportamento, observase que certas condições de existência parecem conferir chance adicional para a ocorrência de enfermidades. Em particular, a condição de depressão materna. 10,18 ou a ocorrência de alcoolismo entre os moradores da casa20,21 conferem à família e, em especial, às crianças em idade pré-escolar, uma situação especial de risco. Muitas vezes, essas condições dificultam ou impedem que as crianças recebam os cuidados necessários para uma situação satisfatória de saúde e de desenvolvimento. Levy, 15 estudando o desenvolvimento estatural de crianças na faixa etária de pré-escolares, enfatizou a importância do ambiente psicosocial onde se dá o crescimento da criança. Neste estudo, as doenças maternas físicas incapacitantes e psicológicas, ocorridas no período pré-escolar dos filhos, mostraramse associadas ao déficit de crescimento. Drotar 21 referiu que famílias com conflitos parentais e mães mais estressadas psicologicamente influenciam as condições de saúde da criança, uma vez que as influências familiares no estado nutricional da criança, por exemplo, não se restringem somente aos efeitos de disponibilidade de alimentos, mas aos modelos de relacionamento familiar, como as crises e as desorganizações presentes entre os membros familiares. Isto é, o padrão disfuncional de relacionamento familiar leva às dificuldades alimentares 
e ingestão calórica inadequada.

O comprometimento das relações entre mães deprimidas e suas crianças, na idade pré-escolar ou escolar, também tem sido documentado por Weiss e Chen, 16 Bifulco et al. ${ }^{17}$ e Hammen e Brennan. ${ }^{18}$ Segundo esses autores, há associação entre saúde mental da mãe e qualidade da interação mãe/criança. Precárias condições emocionais maternas podem levar a um padrão geral de cuidado infantil inadequado, que aumenta o risco para a ocorrência de doenças, desnutrição10 ou retardo de desenvolvimento. 15 Em seu estudo sobre desnutrição em crianças na idade pré-escolar, Carvalhaes e Benício10 identificaram como fator de risco a precária saúde mental materna. Segundo esses os autores, comprometimentos menos evidentes da saúde mental materna, ainda que não-incapacitantes para o cuidado infantil, poderiam elevar o risco para alguns desfechos negativos para saúde.

O alcoolismo, por sua vez, está comumente associado com altos níveis de violência e baixos níveis de afeto no contexto familiar. Quando membros da família fazem uso abusivo do álcool, verificam-se com freqüência episódios em que os adultos não só se agridem mutuamente, mas também são menos afetivos com as suas crianças, além de despenderem menos tempo com os cuidados que elas necessitam.22 Roque e Ferriane, 19 em um estudo abordando a presença de alcoolismo e a interação familiar, identificou que o relacionamento dos pais com os filhos foi considerado ruim ou com algum grau de dificuldade em $78,4 \%$ dos casos. Os problemas mais relatados foram os de comportamento da criança (agressividade, isolamento), e uso de álcool e droga pelos filhos.

Monteiro et al.,23 estudando aspectos psicosociais de pais agressores, afirmam que mães etilistas, provenientes de lares desajustados, têm filhos que podem apresentar inúmeras seqüelas, tais como: desajustamento social, baixa auto-estima, traços de personalidade depressivos e problemas cognitivos, entre outros. Pode-se dizer que há consequiências desfavoráveis, tanto para a saúde física quanto para o desenvolvimento psicosocial de crianças, quando há ocorrência de alcoolismo na família. Em idade pré-escolar, dada a grande vulnerabilidade das crianças, o sofrimento é maior com as disfunções familiares causadas pelo uso abusivo do álcool.

Entende-se que na faixa etária de dois e três anos a influência do cuidado materno sobre a ocorrência da cárie dental se dá com grande intensidade. 24 Considera-se que a partir desse período de vida, de forma crescente, outros fatores passam a exercer influência concorrente com o papel da mãe, no esta- belecimento de padrões de auto-cuidado, conferindo à criança certa autonomia em relação aos papéis maternos básicos.

Uma situação de comprometimento da estrutura familiar pode afetar negativamente a ação da mãe para prover os cuidados básicos com a criança, incluindo aqueles relacionados com os fatores moduladores da cárie. Isto é, a administração de uma dieta saudável, bem balanceada e variada é condição essencial para uma boa saúde geral da criança e para a formação e manutenção de dentes saudáveis. 25 Essas escolhas não raramente recaem sobre a mãe, que também se encarrega de evitar a introdução de hábitos nocivos como chupetas adocicadas, as quais atuam como calmantes imediatos da criança. Essa postura requer decisões firmes e difíceis, para as quais a mãe precisa do apoio daqueles que lhe são próximos e de um estado psicológico positivo para manter este propósito, mesmo com as demandas incessantes, tão comuns nesta faixa etária.

Além disso, o procedimento principal para reduzir a incidência da cárie dental é o estabelecimento de práticas de higiene bucal desde a erupção das primeiras unidades dentárias. Todavia, a realização da limpeza da cavidade bucal da criança após cada ingesta pode ser considerada uma tarefa trabalhosa, principalmente nos casos de crianças que se alimentam imediatamente antes e durante o período de sono. É plausível considerar-se então que a adoção de tais hábitos pode ser fortemente influenciada pela condição materna e ambiência familiar.

Vale notar, entretanto, que os achados desta pesquisa devem ser analisados com cautela. Isso se deve, principalmente, ao pequeno tamanho da população de estudo e ao processo de seleção da mesma, baseado em critérios de conveniência, por se tratar de uma única área geográfica de Salvador. Neste sentido, não se pode descartar os possíveis vieses de seleção, visto que foi impossível a entrevista ou exame da população que recusou a pesquisa, que se constituiu de $30 \%$ do total de crianças da área. O reduzido poder impôs limites à realização da análise multivariada, impedindo a verificação da ocorrência de potenciais interações e dificultando a identificação de fatores de confusão. Desta forma, a apreensão da complexidade de que se reveste a questão abordada ficou, de certa forma, comprometida. Outro aspecto a ser considerado diz respeito ao caráter transversal do estudo, cujos limites são conhecidos para o teste de hipóteses causais, já que não é possível garantir-se a antecedência temporal da exposição em relação ao efeito. A medida da exposição através do indicador proposto também apresenta limites, já que o componente relativo à 
presença de alcoolismo na família foi obtido a partir da percepção da mãe e não da aplicação da escala CAGE a todos os membros da família.

Apesar dos seus limites, os achados deste estudo sugerem que a presença de fatores que comprometem a capacidade materna de cuidar e o equilíbrio do ambiente familiar podem estar associados ao desenvolvimento precoce da cárie dentária e reforçam a necessidade de adoção deste enfoque em outras investigações, através da utilização de metodologia apropriada que supere as limitações aqui apresentadas.

Há que se considerar também que este tipo de abordagem encontra-se em consonância com o

\section{Referências}

1. Tomita NE, Sheiham A, Bijella VT, Franco LJ. Relação entre determinantes socioeconômicos e hábitos bucais de risco para más-oclusões em pré-escolares. Pesq Odontol Bras. 2000; 14: 169-75.

2. Moura LFDA, Moura MS, Toledo AO. Dental caries in children that participated in a dental program providing mother and children care. J App Oral Sci. 2006; 14: 53-60.

3. Peres KGA, Bastos Jr M, Latorre MRDO. Severidade de cárie em crianças e relação com aspectos sociais e comportamentais. Rev Saúde Pública. 2000; 34: 402-8.

4. Murray JJ. Comments on the Conference: Second International Conference on Declining Caries. Int Dent J. 1994; 44 (Suppl 1): 457-8.

5. Ministério da Saúde. Secretaria de Atenção à Saúde. Departamento de Atenção Básica. Coordenação Nacional de Saúde Bucal. Projeto SB Brasil 2003. Condições de saúde bucal da população brasileira 2002-2003: resultados principais. Brasília (DF); 2004.

6. Narvai PC, Biazevic MGH, Junqueira SR, Pontes ERCJ. Diagnóstico da cárie dentária: comparação dos resultados de três levantamentos numa mesma população. Rev Bras Epidemiol. 2001; 4: 72-80.

7. Saito SK, Deccico HMU, Santos MN. Efeito da prática de alimentação infantil e de fatores associados a ocorrência de cárie dental em pré-escolares de 18 a 48 meses. Rev Odontol Univ São Paulo. 1999; 13: 5-11.

8. Petrini JC. Modernidade: entre civilização e barbárie. Algumas repercussões na família. Interfaces: Rev Psicol. 1999; 2: 7-14.

9. Vasconcelos EM. A priorização da família nas políticas de saúde. Saúde Debate. 1999; 23: 6-19.

10. Carvalhaes MABL, Benício MHD. Capacidade materna de cuidar e desnutrição infantil. Rev Saúde Pública. 2002; 36 : 188-97.

11. Manfredini MA. Planejamento em saúde bucal. In: Pereira AC. Odontologia em saúde coletiva: planejando ações e promovendo saúde. Porto Alegre: Artmed; 2003. p. 53-65. momento atual de reordenamento da atenção básica ao nível do Sistema Único de Saúde (SUS) e ampla utilização da estratégia do Programa de Saúde da Família. Segundo Vasconcelos, ${ }^{9}$ recentes estudos sobre a família brasileira revelam que, em alguns casos, nas classes populares, as famílias vivem em situações especiais de risco, o que as torna incapazes de articular minimamente os cuidados de seus membros e por isso necessitam de atenção diferenciada do Estado para garantir os direitos de cidadania das crianças, idosos e deficientes físicos ali presentes. Cabe aos serviços de saúde desenvolver estratégias de prevenção e controle de riscos à saúde através da identificação precoce desses casos.

12. WHO (World Health Organization). Oral health surveys, basic methods. 4. ed. Geneva; 1997.

13. WHO (World Health Organization). Division of Mental Health. A user's guide to the self reporting questionnaire (SRQ). Geneva; 1994.

14. Barros SG, Castro Alves A, Pugliese LS, Reis SRA. Contribuição ao estudo da cárie dentária em crianças de 030 meses. Pesq Odontol Bras. 2001; 15: 215-22.

15. Levy MSF. Relação entre situação social e crescimento físico numa população infantil de Santo André, São Paulo, Brasil. Rev Saúde Pública. 1977; 11: 295-321.

16. Weiss SJ, Chen JL. Factors influencing maternal mental health and family functioning during the low birthweight infant's first year of life. J Pediatric Nur. 2002; 17: 114-25.

17. Bifulco A, Moran PM, Ball C, Jacobs C, Baines R, Bunn A, Wolfgang GM. Chilhood adversity, parental vulnerability and disorder: examining inter-generational transmission of risk. J Child Psycol Psychiatr. 2002; 43: 1075-86.

18. Hammen C, Brennan PA. Interpersonal dysfunction in depressed women: impairments independent of depressive symptoms. J Affect Dis. 2000; 72: 145-56.

19. Roque EMST, Ferriani MGC. Desvendando a violência doméstica contra crianças e adolescentes sob a ótica dos operadores do direito na comarca de Jardinópolis, São Paulo. Rev Latino-Am Enfermagem. 2002; 10: 334-44.

20. Frank DA, Brown J, Johnson S, Cabral H. Forgotten fathers: an exploratory study of mothers'report of drug and alcohol problems among fathers of urban newborns. Neurotoxicol Teratol. 2002; 24: 339-47.

21. Drotar D. Relating parent and family functioning to the psychological adjustment of children with chronic health conditions: what have we learned? What do we need to know? J Pediatr Psychol. 1997; 22: 329-44.

22. Shuntich RJ, Loh D, Katz D. Some relationships among affection, agression and alcohol abuse in the family setting. Percep Mot Skills. 1998; 86: 1051-60. 
23. Monteiro MCN, Cabral MA, Morgado AF. Aspectos psicosociais de pais agressores. J Bras. Psiquiatr. 1995; 44: 7-13.
24. Ramos BC, Maia LC. Cárie, tipo mamadeira e a importância da promoção de saúde bucal em crianças de 0 a

4 anos. Rev Odontol São Paulo. 1999; 13: 39-43.

Recebido em 3 de maio de 2005

Versão final apresentada em 2 de junho de 2006

Aprovado em 27 de julho de 2006 